\title{
DISTRIBUTED LASER THERAPY SYSTEM
}

\author{
Vladimir Ranđelović*, \\ Aleksandar Jevremović \\ ${ }^{1}$ Faculty of Informatics and Computing, \\ Singidunum University, \\ Belgrade, Serbia
}

\begin{abstract}
:
A distributed laser therapy system has been designed to allow laser practitioners to carry out low-level laser therapy (LLLT) and provide simple means to easily collect different laser therapy protocols along with the estimation of the therapy outcome. This article aims to present both the hardware and software design of the system and to discuss three different neural networks used to predict the outcome of the laser therapy, to recommend the laser parameters and to recognize the biological effects. It is concluded that the proposed system could successfully be applied for the collection of a large number of therapy protocols that could later be used for neural network training. Also, the trained neural networks could be easily employed to support the everyday work of laser therapy practitioners by providing different types of laser therapy recommendations.
\end{abstract}

\section{Keywords:}

laser therapy, biomedical equipment, internet of things, neural network, artificial intelligence.

\section{INTRODUCTION}

Laser therapy is a medical procedure which utilizes the healing characteristics of the laser light to treat different types of ailments in physical medicine, rheumatology, dermatology, or neurology, having the following primary biological effects: [1-8]:

- Anti-inflammatory and anti-edematous effect

- Cicatrization, collagen production, wound healing

- Stimulation or inhibition of the cell proliferation

- Normalization of the membrane and action potentials

All the mentioned effects are primarily based on the specific laser light characteristics which include: monochromaticity, coherence, polarization as well as focusability of the laser beam to the very high intensities. Besides the inherent laser characteristics, laser therapy may be characterized by adjustable laser therapy parameters which, in turn, may be used to give rise to different biological effects.

\section{Correspondence:}

Vladimir Ranđelović

e-mail:

vladimir@artmedico.com 
These parameters include energy, power, intensity, and modulation frequency. Example of the laser parameter values, excerpted from the meta-analysis of the published articles [1-8] may be seen in TABLE I.

Since laser therapy is a method daily applied by different laser practitioners, many of whom try to improve therapy results by adjusting laser parameter values, it was though out to design the laser therapy system capable to collect all therapy protocols applied by many different laser therapy practitioners. Data collected likewise could be used to train different types of artificial neural networks (ANN) to help practitioners determine the proper combination of laser parameters, or to differentiate the successful from the unsuccessful therapy protocol.

Table 1. Values of the laser therapy parameters for different indications

\begin{tabular}{lcccc}
\hline $\begin{array}{c}\text { Therapy } \\
\text { parameter }\end{array}$ & Unit & Alveolitis & Cervicalgia & Acupuncture \\
\hline Energy & $\mathrm{J}$ & 1.5 & 2 & 0.5 \\
\hline Power & $\mathrm{mW}$ & 30 & 50 & 3 \\
\hline Intensity & $\mathrm{mW} / \mathrm{cm}^{2}$ & 120 & 120 & 12 \\
\hline Frequency & $\mathrm{Hz}$ & 250 & 2000 & 20 \\
\hline Outcome & & 0.1 & 0.6 & 0.8 \\
\hline
\end{tabular}

Even though ANN are widely used in the biomedical applications, it is only a small number of articles in the literature that deal with ANN related to the biomedical lasers of different types. Majority of those, try to predict the outcome of a single therapy procedure $[9,10]$ based on the patients' characteristics, while some of the articles use ANN as a controller [11], or as a therapy assistance tool for the recognition of the therapy significant events or objects. By reviewing the literature, no articles could be found to deal with the ANN in the field of low-level laser therapy (LLLT) that, based on a large number of collected protocols, try to predict either the therapy outcome or the biological effect on the bases of the chosen therapy parameters, or to propose laser parameters on the bases of the selected indication.

\section{The aim}

The aim of the article is to present the design of the distributed laser therapy system which allows the collection of a large amount of applied laser therapy protocols along with the therapy outcomes. Besides, the aim of the article is to analyze and present the architecture of the neural networks suitable for prediction of the laser therapy outcome, recommendation of the laser parameter values, and recognition of the biological effects.

\section{DESIGN OF THE DISTRIBUTED LASER THERAPY SYSTEM}

Distributed laser therapy system is aimed to allow the practitioner to:

- Use the laser therapy unit reliably and safely on the basis of all applicable biomedical standards.

- Allow connection of all laser therapy units with the central server and let each of the units transfer the values for the applied therapy protocols along with the outcome of the therapy, but also, to allow the laser units to receive therapy recommendations and predictions from the trained neural networks located in the central server.

- Make possible the proper training of neural networks with a large number of therapy protocols obtained from different laser therapy practitioners, as well as to provide the storage of the trained neural network models in the database, and to allow the usage of the trained models in order to get therapy recommendations, predictions of the therapy outcomes, and recognition of the biological effects.

The design of the system includes the system topology, the laser probe, as well as all the software components that take part in the system:

- Laser probe software installed within the microcontroller circuit of the probe.

- Desktop application used by practitioners to control the laser probe and to communicate with the central server in order to send the applied therapy protocols, and to get therapy recommendations from the neural networks operating within the server.

- Server application, the role of which is to communicate with all laser units as well as to host the neural networks used to provide therapy recommendations.

- Database used to store all data related to different users, patients, therapy protocols and trained models of neural networks.

\section{Topology}

The topology of the distributed laser therapy system is presented in Fig. 1. Any laser treatment facility could be equipped with at least one of these laser therapy units and, over the internet, could be connected with the application server. This way, each of the therapy units can send applied therapy protocols to the server. 


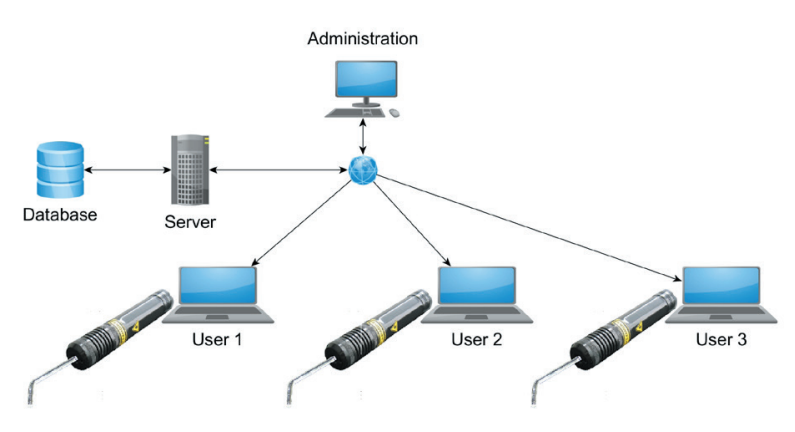

Fig. 1. The topology of the distributed laser therapy system

The application server is directly connected to the database server both of which could physically be located in the same or separate machines.

\section{Applicable standards}

During the system design, all applicable biomedical standards were taken care of. These include general medical devices standard IEC60601-1 [12], as well as standards for medical laser equipment IEC 60601-2-22 [13]. Also, during the laser operation, all proposed safety precautions for $3 \mathrm{~B}$ class lasers were taken into account. As defined by the standards, these included the safety glasses with appropriate spectral characteristics, as well as safety switches.

\section{Laser probe}

The hardware part of the system is composed of the laser probe (Fig. 2, Fig. 3), connected to the computer (Fig. 4) over the USB port. Inside the probe, there is the printed circuit board (PCB) with microcontroller and electronic components necessary for the laser irradiation control. PCB is connected with the laser diode which directly adheres to the light guide (Fig. 2).

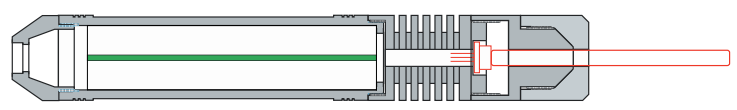

Fig. 2. Laser probe cross-section

Light guides are used to deliver laser light to both accessible and inaccessible parts of the body without energy loss, maintaining constant intensity at the contact surface. Given the fact that they get in contact with the patient's tissue, they need to be replaced and sterilized after each application.
The probe is equipped with the light guide mounting system [14] which allows easy attachment and detachment of the light guides.

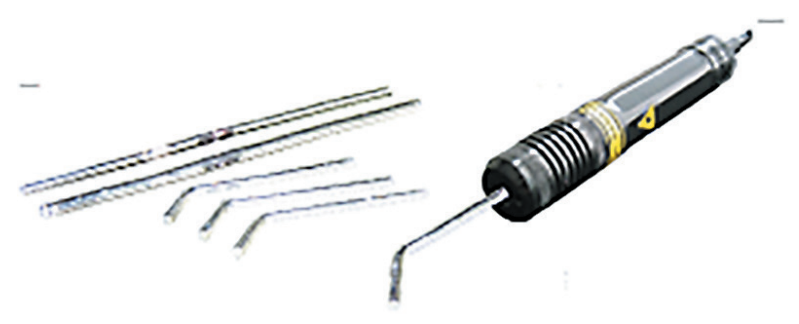

Fig. 3. Laser probe with light guides

Central component of the laser unit is the laser probe which, for the laser therapy purposes, usually uses laser diodes that generate laser light with wavelengths between $780-904 \mathrm{~nm}$. The laser diode is a very sensitive and expensive component which requires an electric connection over the driving circuits to guarantee its reliable operation.

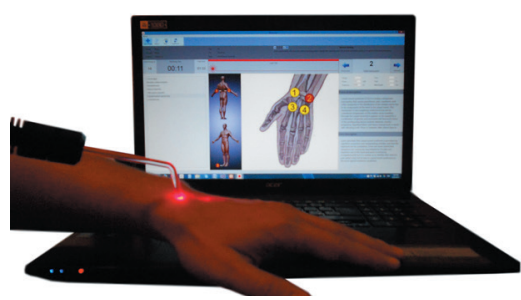

Fig. 4. Laser therapy unit

The laser probe is connected to the personal computer which requires the desktop application designed to communicate with the probe microcontroller and transfer all the therapy parameters. At the beginning of therapy, microcontroller receives the laser parameters from the desktop application over the USB port (Fig. 5 ), and the diver module, over DA (digital to analog) converters, control lines, and internal signal generator, drives the analog laser lines, while simultaneously, over control lines and AD (analog to digital) converter, it reads the state of the laser diode in order to allow driver module to maintain irradiation at the level defined by the laser parameters [15].

On one side, the purpose of the analog laser control is to maintain irradiation parameters at the specified 
level, while on the other side, its purpose is to filter all electrical disturbances that could potentially reach the diode and damage it. Laser diode is an expensive element very sensitive to transient electrical surges.

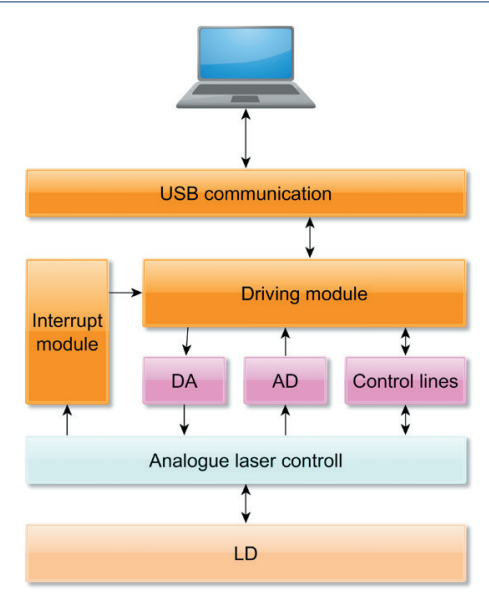

Fig. 5. The architecture of the microcontroller application and connection with the analog laser control

Having in mind the limitations of the laser therapy defined in the literature and the limitations of the safety class 3B lasers, the laser probe was constructed to fulfill these requirements and has the characteristics presented in TABLE II.

Table 2. Values of the laser therapy parameters for different indications

\begin{tabular}{lcc}
\hline \multicolumn{1}{c}{ Therapy parameter } & Unit & Value range \\
\hline Energy & $\mathrm{J}$ & $0-10$ \\
\hline Power & $\mathrm{mW}$ & $0-100$ \\
\hline Intensity & $\mathrm{mW} / \mathrm{cm}^{2}$ & $0-400$ \\
\hline Frequency & $\mathrm{Hz}$ & $0-10000$ \\
\hline
\end{tabular}

\section{Desktop application}

Besides the hardware components, the most important part of the system for carrying out the therapy surely is the desktop application. Its main purpose is to control the work of the laser probe and communicate with the server in order to transfer all the applied therapy protocols, and also to receive the therapy recommendations from the trained neural networks. Desktop application is realized using the C\# in Visual Studio 2013. It allows the practitioner to select the therapy protocol and, on its initiation, transfer the laser parameters to the microcontroller application.

After starting the application, the available therapy protocols are first synchronized with the server and then presented to the practitioner. The synchronization is started in the presentation layer (Fig. 6), which calls the service layer in charge for the communication. On the server side, the web layer receives the request and engages the subsequent layers to retrieve all the available therapy protocols from the database, and then returns them back to the desktop application.

The sequence of events engaged to start the laser therapy is initiated in the presentation layer of the desktop application (Fig. 6) which further calls the communication layer, over the service layer, to transfer the therapy parameters to the laser probe and start the therapy. In the further course of the therapy, the microcontroller application monitors and controls the process of irradiation. In case of the laser malfunctioning, or incompliances between the defined and measured laser parameters, the microcontroller application informs the desktop application which, in turn, forwards the information to the practitioner.

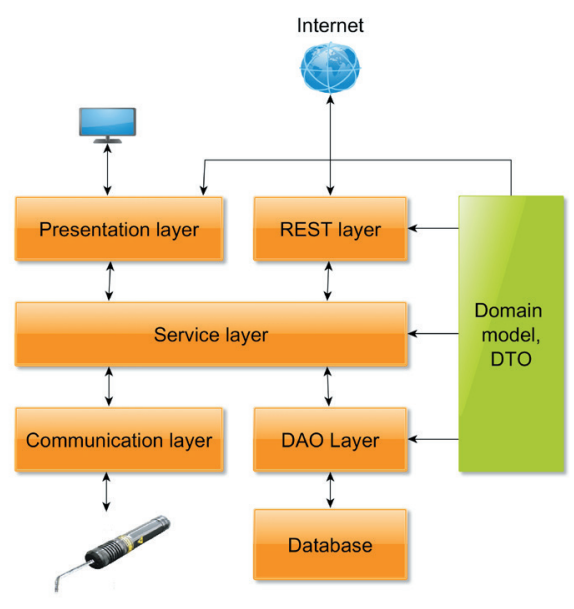

Fig. 6. The desktop application architecture

During the course of the therapy, the practitioner can follow the recommended irradiation points (Fig. 4). Desktop application guides the practitioner to follow the order of the treatment points, while the laser probe automatically receives the laser parameters in the way that practitioner does not have to readjust settings of frequency, energy or power for different points.

\section{Server application}

Server application is created using Java v1.8 language and Spring v4.2.4. framework [16]. Besides Spring, Hibernate v5.1 was used as object-relational mapper and database access tool. 
The architecture of the application (Fig. 7) is based on a multilayer model including the web, service, and DAO (Data Access Object) layers [17].

Web layer was designed to provide REST API, using which, all the desktop applications can communicate with the application server. All requests received in the web layer are realized over the service and DAO layers. The service layer is used to control the training and the operation of the neural networks, while DAO layer is used to provide a connection with the database.

Therefore, in case that the neural network should be trained to recognize the resulting biological effect on the basis of defined laser parameters, the service layer is chosen to host the algorithms for the neural network training, while the trained model is, over DAO layer, stored in the database.



Fig. 7. The server application architecture

Entity and DTO classes are used for the interlayer communication, but also for serialization/deserialization during the communication between the desktop and server applications.

\section{Database}

The database is implemented using the Oracle v11g. As shown in Fig. 8, the database tables are organized in the way that one therapy is connected to many protocols, each of which is in relation to many treatment points. On the other side, practitioners (Users) can access only their own patients, each of which could have multiple therapies. The models of the trained neural networks are stored in the ANN_weights table. Each therapy protocol can be connected with many different types of ANN, each of which contains its own weights defined in the table ANN_weights.

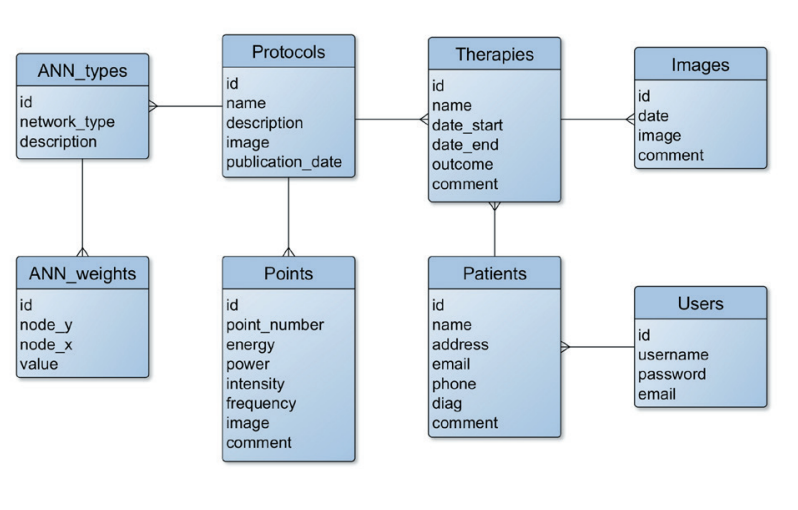

Fig. 8. E/R database model

\section{DATAANALYSIS}

As can be seen in the table (TABLE I. ), each therapy protocol consists of the diagnose and laser parameters such as energy, power, intensity, and frequency. Besides the parameters, each protocol contains the therapy outcome expressed as a number between 0 and 1 , where 0 means completely unsuccessful, while 1 means completely successful therapy.

Protocols stored in the database can be used for different purposes, with the final goal in mind, to make the work of the laser practitioner easier and more successful. Using the community knowledge stored in the database, neural networks could be trained to understand various aspects of this knowledge. Therefore, different modalities of their application will be discussed subsequently:

1. Application of ANN (Artificial Neural Network) for the recognition of the therapy outcome.

2. Application of ANN for the therapy parameter recommendation on the basis of the selected biological effect.

3. Application of ANN for the recognition of the biological effect on the basis of defined laser parameters.

All mentioned ANN are realized in Java v1.8 programming language, regardless of any existing machine-learning library, so that the number of input, hidden and output nodes, as well as the number of hidden layers can be changed dynamically depending on the requirements [18-20].

\section{Therapy outcome estimation}

The evaluation of the therapy outcome is realized using the multilayer perceptron with the architecture shown in Fig. 9.

The network has four input nodes into which the values of laser parameters (energy, power, intensity, and frequency) are fed forward. 
The perceptron has one node in the output layer which gives the prediction for the therapy outcome. Having in mind that the network architecture could be defined dynamically, the network would be able to self-adjust by adding new nodes in the input layer if other laser parameters had to be taken into consideration.

To prepare the network, it is necessary to train it using both the values of the therapy parameters, along with the treatment outcome which, in this case, would be used as a supervised value. The training process is initiated by the system administrator over the server application.

After receiving the training request, the server application retrieves all available protocols from the database and feeds them forward to the neural network. At the same time, therapy outcome related to the current protocol is used as supervised value and the network is trained using the backpropagation technique [21].

This network may be used whenever the practitioner wishes to check for the prediction of the therapy outcome on the basis of the selected therapy parameters. In order to check for the prediction of the therapy outcome, the practitioner first defines the values for each of the therapy parameters (energy, power, intensity, frequency) and asks for the prediction. The desktop application contacts the server application by sending the defined therapy parameters.

The server application loads the trained network model and feeds forward the received values for the therapy parameters. The ANN responds with the prediction in the output layer and the server application returns the prediction to the practitioner. The result is obtained in a range between 0 and 1 , where a number closer to 0 means minimal chance, while a number closer to 1 means maximum chance of having a successful therapy outcome.

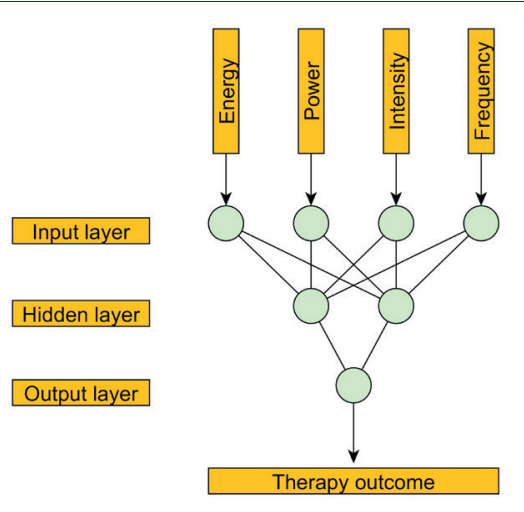

Fig. 9. The architecture of the multilayer perceptron for the therapy outcome prediction
This means that the practitioner can get the prediction of the therapy outcome and decide in advance whether to proceed with it or not. The effectiveness of this ANN is still to be tested on the bases of the real therapy parameters and real therapy outcomes. Once these tests are performed, it would be possible to bring conclusions about the effectiveness of this neural network.

Laser parameter recommendation on the bases of the chosen biological effect

The neural network used for the recommendation of the therapy parameters has the potentiality to be used most frequently simply because this represents the most common request among practitioners. The architecture of the multilayer perceptron is shown in Fig. 10. The ANN has three input nodes that correspond to the number of biological effects, while in the output layer it has the number of nodes that corresponds to the number of laser parameters. In this case, the network has three input nodes and four output nodes. Given the fact that the architecture of the network can be adjusted dynamically, it is not a problem to change it during the run time and retrain the network accordingly. The number of hidden layers and their nodes would be dependent on the experimental results obtained during the perceptron testing. The desired biological effect could simply be selected by feeding forward the value 1 to the input node that corresponds to the chosen effect, while all the other input nodes would be fed forward the value 0 . At its output layer, the values of the corresponding laser parameters (energy, power, intensity, and frequency) are obtained.

This network can be trained the same way as the previous ANN, but this time, only the therapy protocols whose therapy outcome is greater than the defined threshold should be loaded from the database. The higher value the threshold has, the more reliable therapy protocols would be loaded from the database. The protocols selected this way are used for the ANN training in the way that the selected biological effects are fed forward to the input nodes of the network, while the laser parameters are used in the process of backpropagation to adjust the weights. After completing the training, the trained model of the perceptron is stored in the ANN_weights table of the database (Fig. 8). 


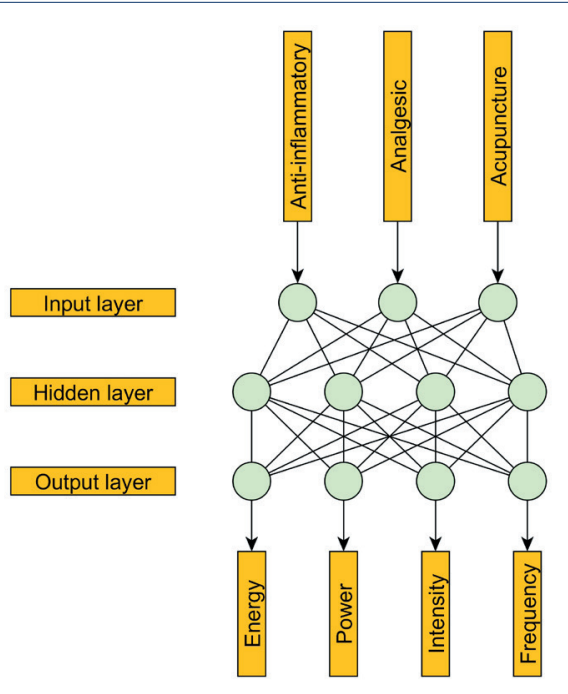

Fig. 10. The architecture of the multilayer perceptron for the laser parameter recommendation on the bases of the chosen biological effect

In daily work, the practitioner can select the desired diagnose or a biological effect in the desktop application and ask for the therapy recommendation. The desktop application would immediately contact the server sending the chosen biological effect, and the server application can load the trained network model from the database and ask for a recommendation. At the output layer, the network would deliver the recommendation for the laser parameters and server would return these values to the desktop application. After reviewing the recommendation, the practitioner may accept the recommended values or not.

In order to check the practical value of the presented ANN, its effectiveness should be investigated with the real laser parameter values and realistic therapy outcomes.

\section{Therapy protocol recognition}

In many occasions, laser therapy practitioners need to understand which therapeutic effect could be achieved by employing certain therapy parameters. To recognize the biological effect on the basis of laser parameters, the multilayer perceptron with the following architecture (Fig. 11) has been created.

The architecture of the ANN is designed in the way that the input layer has as many nodes as there are laser parameters, while the output layer has as many nodes as there are biological effects to be recognized.
To train the network, it is necessary to use only protocols from the database with therapy outcome above a certain threshold. Ideally, only the protocols with maximal therapy outcome should be retrieved. The laser parameters of the protocols are fed forward to the input nodes of the perceptron, while the biological effect is used during the backpropagation in the output layer of ANN by specifying number 1 as the supervised value of the output node of the resulting biological effect and specifying 0 for all the other supervised values.



Fig. 11. Architecture of the multilayer perceptron for the biological effect prediction on the bases of the therapy parameters

By testing this type of neural network [8], it was concluded that in the case of application of therapy protocols employing all four laser parameters used in this article, the network was successful in classifying $100 \%$ of the protocols. That is, the network did not make any mistakes in the recognition of any therapy protocol when all 4 laser parameters (energy, power, intensity, frequency) were specified. In cases of therapy protocols specifying only 3 laser parameters, the success rate ranged from $67-100 \%$.

\section{CONCLUSION}

In reference to the aim of the article, it can be concluded that the distributed laser therapy system is designed in accordance with the following targets:

- The system was designed in accordance to all applicable biomedical standards and is equipped with all the electronic and software means to generate the laser radiation in accordance with 
the laser parameter specification which includes energy, power, intensity, and frequency.

- The system is designed to collect therapy protocols along with the therapy outcomes from all the users of the system.

- The system allows the training, storage, and usage of multilayer perceptrons with the proper architecture for: the estimation of the therapy outcome, the recommendation of the laser parameters on the basis of the chosen biological effect, as well as for the biological effect recognition on the basis of the chosen laser parameters.

It may be concluded that the presented design allows the users to obtain therapy recommendations based on the neural networks trained with the community knowledge of all the users of the distributed laser therapy system.

\section{REFERENCES}

[1] V. Randjelovic., D.Tatic. J.Randjelovic, Computer Controlled Laser, Nis, Ei Bull HN, 1994.

[2] F. Watban, X. Zhang, "The comparison of effects between pulsed and CW lasers on wound healing", JClin Laser Med Surg., vol. 22, no. 1, pp. 15-18, Feb. 2004.

[3] D. Hawkins, H Abrahamse, "Laboratory Methods for Evaluating the Effect of Low Level Laser Therapy (LLLT) In Wound Healing", African Journal of Biomedical Research, vol. 8, pp.1-14, 2005,

[4] JH. Lee, MH. Chiang, PH. Chen, ML. Ho, HE. Lee, YH. Wang, "Anti-inflammatory effects of low-level laser therapy on human periodontal ligament cells: in vitro study", Lasers Med Sci., vol. 33, no. 3, pp. 469-477, 2018.

[5] A.Alves, R.Vieira, E. Leal-Junior, S. Santos, A.Ligeiro, R. Albertini, J. Silva Junior, P. de Carvalho, "Effect of low-level laser therapy on the expression of inflammatory mediators and on neutrophils and macrophages in acute joint inflammation", $A r$ thritis Res Ther., vol. 15, no. 5, 2013.

[6] M. Artés-Ribas, J. Arnabat, A. Puigdollers, "Analgesic effect of a low-level laser therapy $(830 \mathrm{~nm})$ in early orthodontic treatment", Lasers in Medical Science, vol. 28, no. 1, 2012.

[7] S. Moskvin, "Low-Level Laser Therapy in Russia: History, Science and Practice", J Lasers Med Sci., vol. 8, no. 2, pp. 56-65, 2017.

[8] V. Randjelovic, "IoT Based Laser Therapy System and Multilayer Perceptron for the Recognition of the Therapy Protocols", Telsiks conference proceedings, 2019, in press.
[9] A. Nagy, Z. Mowafy, R. Alkhoribi, "Prediction of abdominal contouring response to low level laser using Artificial Neural Network", Bioscience Research, 2018; 15(2): 950-955.

[10] S. Cazzaniga, F. Sassi, S. Mercuri, L. Naldi, "Prediction of clinical response to excimer laser treatment in vitiligo by using neural network models", Dermatology. 2009;219(2):133-137.

[11] S. Razavi, "A neural controller for online laser power adjustment during the heat therapy process in the presence of nanoparticles", Australas Phys Eng Sci Med. 2017;40(2):401-411.

[12] IEC standard: Medical Electrical Equipment, IEC60601-1, 2000.

[13] IEC standard: Particular requirements for basic safety and essential performance of surgical, cosmetic, therapeutic and diagnostic laser equipment, IEC 60601-2-22, 2007.

[14] V. Randjelovic., Patent: Light guide holder for laser probe, Intellectual property Office of Serbia: MP836/2006.

[15] V. Randjelovic., Patent: USB therapy laser, Intellectual property Office of Serbia: MP837/2006.

[16] W. Wheeler, J. White, "Spring in Practice“, Shelter Island: Manning, 2013.

[17] C. Binildas, B. Malhar, C. Vincenzo, "Service Oriented Architecture with Java", Birmingham: Packt Publishing, 2008.

[18] M. Kubat, "An Introduction to Machine Learning", New York, Springer, 2015.

[19] J. Bell, "Machine Learning“, John Wiley and Sons: Indianapolis, 2015.

[20] F.M.Soares, A.M. Souza, "Neural Network Programming with Java", Packt Publishing: Birmingham, 2016.

[21] M. Buscema, "Back Propagation Neural Networks", Subst Use Misuse, vol. 33, no. 2, pp. 233-70, 1998. 